\title{
How to Obtain a Mass of a Graviton, and Does This Methodology Lead to Voids?
}

\author{
Andrew Walcott Beckwith \\ Department of Physics, PRC (Visiting Scholar) Chongqing University, Chongqing, China \\ Email: Rwill9955b@gmail.com, abeckwith@uh.edu
}

How to cite this paper: Beckwith, A.W. (2020) How to Obtain a Mass of a Graviton, and Does This Methodology Lead to Voids? Journal of High Energy Physics, Gravitation and Cosmology, 6, 416-439. https://doi.org/10.4236/jhepgc.2020.63032

Received: June 13, 2020

Accepted: July 14, 2020

Published: July 17, 2020

Copyright $\odot 2020$ by author(s) and Scientific Research Publishing Inc. This work is licensed under the Creative Commons Attribution International License (CC BY 4.0).

http://creativecommons.org/licenses/by/4.0/ (c) (i) Open Access

\begin{abstract}
Using the Klauder enhanced quantization as a way to specify the cosmological constant as a baseline for the mass of a graviton, we eventually come up and then we will go to the relationship of a Planck Length to a De Broglie length in order to link how we construct a massive graviton mass, with cosmological constant and to interface that with entropy in the early universe. We then close with a reference to the possible quantum origins of e folding and inflation. This objective once achieved is connected with a possible mechanism for the creation of voids, in the later universe, using a construction of shock fronts from J. P. Onstriker, 1991 and followed up afterwards with Mukhanov's physical foundations to Cosmology book section as to indicate how variable input into self reproduction of the Universe structures may lead to void formation in the present era. A connection with Wesson's 5 dimensional cosmology is brought up in terms of a generalized uncertainty principle which may lead to variations of varying energy input into self reproducing cosmological structures which could enable non uniform structure formation and hence voids. One of the stunning results is that the figure of number of gravitons, about $10^{58}$, early on, is commensurate with a need for negative pressure, (middle of manuscript) which is a stunning result, partly based on Volovik and weakly interacting Bose gas model for pressure, which is completely unexpected. Note that in quantum physics, the idea statistically is that at large quantum numbers, we have an approach to classical physics results. We will do the same as to our cosmological work. This means that the $n_{\text {quantum number }}^{2} \gg 1$, in our last set of equations, which as we indicate has the surprise condition that for Pre-Planckian space-time that a very large value for initial Pre Planckian dimensions $d_{\text {dim }}$ which is the dimensional input into the Pre Planckian state, prior to emergence into Planckian cosmology conditions. We conclude by stating the following question. Can extra dimensions come from a Multiverse feed into Pre-Planckian space-time? See Theo-
\end{abstract}


rem at the end of this publication. Our answer is in the affirmative, and it has intellectual similarities to George Chapline's work with Black hole physics.

\section{Keywords}

Black Holes, Enhanced Quantization, Gravitons, Voids

\section{Start with the General Relativity First Integral}

We use the Padmanabhan $1^{\text {st }}$ integral [1], of the form, with the third entry of Equation (1) having a Ricci scalar defined via [2] and usually the curvature $\aleph$ is set as extremely small, with the general relativity [3] [4]

$$
\begin{gathered}
S_{1}=\frac{1}{2 \kappa} \cdot \int \sqrt{-g} \cdot \mathrm{d}^{4} x(\Re-2 \Lambda) \\
g=-\operatorname{det} g_{u v} \\
\Re=6 \cdot\left(\frac{\ddot{a}}{a}+\left(\frac{\dot{a}}{a}\right)^{2}+\frac{\aleph}{a^{2}}\right)
\end{gathered}
$$

Also, the variation $\delta g_{t t} \approx a_{\min }^{2} \phi$ by [5] [6] will have an inflaton, $\phi$ given by [7]. Leading to the inflaton which is combined into other procedures for a solution to the cosmological constant problem. Here $a_{\min }$ is a minimum value of the scale factor and is not zero, but close to it.

\section{Next for the Idea from Klauder}

We are going to go to page 78 by Klauder [4] of what he calls on page 78 a restricted Quantum action principle which he writes as $S_{2}$ and we write a 1-1 equivalence as in [1], which is also seen in [3]

$$
S_{1}=\frac{1}{2 \kappa} \cdot \int \sqrt{-g} \cdot \mathrm{d}^{4} x(\Re-2 \Lambda) \approx S_{2} \equiv \int_{0}^{T} \mathrm{~d} t \cdot\left(p(t) \cdot \dot{q}(t)-H_{\tilde{N}}(p, \dot{q})\right)
$$

where

$$
\Re=6 \cdot\left(\frac{\ddot{a}}{a}+\left(\frac{\dot{a}}{a}\right)^{2}+\frac{\aleph}{a^{2}}\right)
$$

Our assumption is that $\Lambda$ is a constant, hence we assume then the following approximation, from [3] which is the precursor of activity as given in [4] [5] [6] [7] we have

$$
\begin{gathered}
\frac{p_{0}^{2}}{2}=\frac{p_{0}^{2}(\tilde{N})}{2}+\tilde{N} \text { for } 0 \leq \tilde{N} \leq \infty, q=q_{0} \pm p_{0} \cdot t \\
V_{\tilde{N}}(x)=0, \text { for } 0<x<1 \\
V_{\tilde{N}}(x)=\tilde{N}, \text { otherwise } \\
H_{\tilde{N}}(p(t), q(t))=\frac{p_{0}^{2}(\tilde{N})}{2}+\frac{(\hbar \cdot \pi)^{2}}{2}+\tilde{N} \text { for } 0 \leq \tilde{N} \leq \infty
\end{gathered}
$$


Our innovation is to then set $q=q_{0} \pm p_{0} t \approx \phi$ and assume small time step values. Then as in [7]

$$
\Lambda \approx-\kappa \cdot \frac{\left[\frac{V_{0}}{3 \gamma-1}+2 \tilde{N}+\frac{\gamma \cdot(3 \gamma-1)}{8 \pi G \tilde{t}^{2}}\right]}{\int \sqrt{-g} \mathrm{~d}^{3} x}+6 \cdot\left(\frac{\ddot{a}}{a}+\left(\frac{\dot{a}}{a}\right)^{2}\right)
$$

These are terms within the bubble of space-time given in [1] using the same inflaton potential. The scale factor is presumed here to obey the value of the scale factor in [8].

\section{Why This Is Linked to Gravity/Massive Gravitons, and Possibly Early Universe Entropy}

Klauder's program [4] is to embed via Equation (3) as a quantum mechanical well for a Pre-Planckian-system for inflaton physics as given by Equation (3). And Equation (3a) and Equation (3b) as given in Klauder's treatment of the action integral as of page 87 of [4] where Klauder talks of the weak correspondence principle, where an enhanced classical Hamiltonian, is given 1-1 correspondence with quantum effects, in a non-vanishing fashion. If so, by Novello [9] and Equation (3) and Equation (3a) and Equation (3b), we have then for early universe conditions, that we will be leading up to using an algorithm for massive gravitons, as in [7], and [9]. If so then use Equation (4) and then get the mass of a graviton via

$$
m_{g}=\frac{\hbar \cdot \sqrt{\Lambda}}{c}
$$

The long and short of it is, to tie this value of the cosmological constant, and the production of gravitons due to early universe conditions, to a relationship between De Broglie wavelength, Planck length, and if the velocity v gets to a partial value close to the speed of light, that, we have, say by using [10] as given by Diosi, in Dice (2018) for quantum systems, if we have instead of a velocity much smaller than the speed of light, a situation where the particle moves very quickly (a fraction of the speed of light) that instead of the slow massive particle postulated in [10]

$$
\begin{gathered}
\lambda_{\text {De Broglie }} \approx \frac{2 \cdot \pi \cdot \hbar}{m_{g} v(\text { velocity })} \cdot \sqrt{1-\left(\frac{v(\text { velocity })}{c}\right)^{2}} \simeq \ell_{\text {Planck }} \\
\ell_{\text {Planck }}=\sqrt{\frac{\hbar G}{c^{3}}}
\end{gathered}
$$

If the velocity of particle is just under the speed of light, i.e. $v$ (velocity) $\rightarrow c-\delta^{+}$ one then would have

$$
\varepsilon(\text { Particle energy }) \approx E_{\text {Planck }}(\text { Planck energy })
$$

If so then, we will be looking at using $\mathrm{Ng}$ version of entropy via use of infinite quantum Statistics [11], we have for a clearly specified value of mass of the gra- 
viton, as in [12], then we have for the negative components. Set here, $c=1, m_{g} \approx 10^{-62}$ grams, as in [12] for the mass of a massive graviton and

$$
E_{\text {Planck }}(\text { Planck energy }) \simeq 2.18 \times 10^{-5} \text { grams }
$$

$$
E_{\text {Planck }}(\text { Planck energy }) \simeq 2.18 \times 10^{-5} \text { grams } \approx m_{g} \times N(\text { Entropy count })
$$

Then we have a superscript $\mathbb{N}$ which has about the numerical number of the e folds as given next, due to

$$
N(\text { Entropy-count }) \simeq 10^{58} \equiv 10^{\mathbb{N}} .
$$

\section{Can This Tie in with Early Universe E Folds ? i.e. from [13] E Folds Are between 55 to 60}

E folds in cosmology are a way of delineating if we have enough expansion of the universe is in line with inflation. In order to solve the most important cosmological problems. As seen in [10] we can have inflation.

$$
\mathbb{N}(\text { e-folding })=-\int \mathrm{d} t H(\cos m)
$$

Here, $H(\cos m)$ a value of the Friedman equation, and if we use [12] be defined via that the potential energy, $V$, of initial inflation is initially over shadowed by the contributions of the Friedman equation, $H$, at the onset of inflation.

Then

$$
\mathbb{N}(\text { e-folding })=55-60
$$

What we wish to explore will be if Equation (9) above is consistent with

$$
N(\text { Entropy }) \approx 10^{58} \equiv 10^{\mathbb{N}} \Leftrightarrow \mathbb{N} \approx 58
$$

Doing so may involve use of the Corda article, as given in [11].

\section{Now for Foundational Treatment as to if We May Have an Influence of the $5^{\text {th }}$ Dimension in Our Problem}

Wesson, [12] has a procedure as far as a five-dimensional uncertainty principle which is written as, if $n \sim L / l$.

Where $L$ is for $4^{\text {th }}$ dimensions, and $l$ is a five-dimensional representation, so we have

$$
\mathrm{d} S_{5}^{2}=n^{2} \mathrm{~d} s_{4}^{2}-n^{4} \mathrm{~d} l^{2}=\left[\frac{L}{l}\right]^{2} \mathrm{~d} s_{4}^{2}-\left[\frac{L}{l}\right]^{4} \mathrm{~d} l^{2}
$$

Then we have an uncertainty principle in 5 dimensions as by Wessson [12] for which we can do if we look at the zeroth contribution

$$
\left|\mathrm{d} p_{\alpha} \mathrm{d} x^{\alpha}\right| \equiv \frac{\mathrm{d} n^{2}}{n} \underset{\alpha \rightarrow 0}{\longrightarrow}|\mathrm{d} E \mathrm{~d} t| \approx|\Delta E \Delta t| \equiv \int \frac{\mathrm{d} n^{2}}{n} \equiv n \cdot \ln n
$$

Using an expansion of the form from CRC tables [13]. 


$$
n \ln n=n \cdot(n-1)-\frac{n}{2} \cdot(n-1)^{2}+\frac{n}{3} \cdot(n-1)^{3}+\cdots
$$

Up to cubic roots, we obtain one real root and 2 conjugate complex roots of, if we use minimum uncertainty of $\Delta E \Delta t=\hbar=1$ and set $c=1$, we have then one real root, and two conjugate complex roots, so that

$$
\begin{gathered}
n_{1} \simeq 1.54715 \quad(\text { as real root for a cubic equation for } n) \\
n_{2,3} \simeq 0.426413 \pm 1.2242 i \quad \text { (as two complex conjugate roots for } n \text { ) }
\end{gathered}
$$

If so for the real case, of $n$, we have about the Planckian regime we look at

$$
l=\frac{l_{\text {Planck }}}{1.54715}
$$

We will then look at the consequences of the real root, first, in terms of variation of minimum time step before going to other cases, but for the record, we have then the weird case of, for real root $n$ in Equation (14) that to other cases, but for the record, we have then the weird case of, for real root $n$ in Equation (14) that

$$
\Delta t \approx-\frac{0.845184}{\Delta E}
$$

Equation (16) is real valued only if $\Delta E<0$.

\section{Under What Conditions Is $\Delta E \leq 0$ How Would Negative Energy Tie into Negative Pressure Which Is Normally Expected in the Onset of Inflation?}

First of all, look at conditions for rapid acceleration of the Universe, i.e. to have this according to the GR theory we have by [14] if $a(t)$ is a scale factor, then the Friedman equations read as

$$
\frac{\ddot{a}}{a}=-\frac{4 \pi G}{3} \sum_{j=1}\left(\rho_{j}+3 p_{j}\right)+\frac{\Lambda_{b}}{3}
$$

Then if we go to Gravitons again, and $j=1$ we can state Equation (17)

$$
\underset{j=1, a(t)=a_{M I N} t^{\alpha}}{\longrightarrow} \Lambda_{b}=3 \alpha^{2}-3 \alpha+4 \pi G\left(\rho_{j=1}+3 p_{j=1}\right)
$$

Now, look at a concept of pressure. Here. If the first expression is tabulated about Planck time (or just before).

We can then make the identification that we have negative pressure, we then have if we have both pressure and energy negative then we can make the following pairing of terms, i.e. first for the negative terms in Equation (18) and also looking at Equation (4), for $j=1$, with the value of the cosmological constant in Equation (4) used, with $\Lambda=\Lambda_{b}$ and with negative terms of Equation (4) and Equation (18) used, we have

$$
-\frac{3 p_{\text {momentum }} \kappa}{\int \sqrt{-g} \mathrm{~d}^{4} x}=-3 \alpha+12 \pi G \cdot\left(p_{j=1}(\text { pressure })\right)
$$

Usually, the $\alpha$ is small so then the momentum term is such that the pres- 
sure is negative. As seen in Equation (19), and we furthermore elaborate upon this in the next section, via what is brought up by [14] from Volovok. But before doing this:

We will after this is described go to the positive terms in Equation (18). The density in our problem we assert is due to the same positive terms in Equation (18a) and comparing what we have with Equation (4) if we make the following positive term identification. With $\Lambda=\Lambda_{b}$

$$
\frac{H_{\text {Potential well hamiltonian }}(p(t), q(t)) \kappa}{2 \int \sqrt{-g} \mathrm{~d}^{4} x}+6 \cdot\left(\alpha^{2}+\frac{\aleph}{a_{\text {min }}^{2} t^{2 \alpha}}\right)_{t=t_{\text {Planck }}}=6 \alpha+4 \pi G \cdot\left(\rho_{j=1}\right)
$$

We will then be looking at how we can then equate out a negative energy and a negative pressure for this Pre Planckian to Planckian physics transition.

\section{Explicit Calculation for a Negative Pressure in This Pre-Planckian to Planckian Physics Transition}

We will transition to Reference [14] by Volovik, 2003 which has the following expression for pressure in a vacuumstate of weakly interacting Bose Gases. i.e. We use then Equation (21) for pressure, if we use the following approximations $E_{\text {Planck1 }}=m c^{2}, E_{\text {Planck2 }}=\hbar c /(\Theta=\sqrt[3]{n(\text { particle density })})$, and so then we have a pressure for Bose pressure

$$
{ }^{+} p_{\text {Bose fluid }}(\text { pressure })=\frac{1}{2 \hbar^{3}} \cdot\left(E_{\text {Planck } 2}^{3} E_{\text {Planck } 1}-\frac{16}{15 \pi^{2}} E_{\text {Planck }}^{4}\right)
$$

This expression becomes negative if $E_{\text {Planck } 2}^{3} E_{\text {Planck } 1}<\frac{16}{15 \pi^{2}} E_{\text {Planck1 }}^{4}$ and if so we have negative pressure. i.e. For our problem, if we configure the initial contents of the "well" we assume for having a near singularity, for space-time expansion start we can have $n$ (Particle density) $=N / V$ (volume), with $N$ as the number of would be "gravitons, and $V$ (volume) being the "Volume of space-time for our evaluation". Whereas $m=m($ mass $)=N($ number gravitons $) \times m_{g}$ with $m_{g}=$ mass (heavy gravity graviton). If so a simple calculation for this problem would have, then a negative value for pressure if we have the following, namely

$$
\frac{\hbar c \cdot \sqrt[3]{V_{\text {Volume }}}}{\left(N_{\text {graviton number }} m_{g}\right)^{1 / 3}} \leq \sqrt[3]{\frac{16}{15 \pi^{2}}} \times N_{\text {graviton number }} m_{g} c^{2}
$$

Here, set $m_{\text {graviton }} \leq 10^{-65}$ to $10^{-62}$ grams, from [15], and Planck $m_{\text {Planck }}=2.176 \times 10^{-5}$ grams [16] And then also useAnd then also use $V($ volume $)=$ cube of planck length $\times(0.27002422918)$

Therefore if Planck length $=L_{\text {Planck }}=1=\hbar=m_{g}$, we have Equation (22) re written as

$$
\left(\frac{15 \pi^{2}}{16}\right)^{1 / 3} \times 0.270024 \leq\left(0.5 \times 10^{-58} m_{P l}\right)^{4 / 3} N_{\text {graviton }}^{4 / 3}
$$


Or roughly

$$
\left(\frac{15 \pi^{2}}{16}\right)^{1 / 3} \times 0.270024 \leq 10^{-77} N_{\text {graviton }}^{4 / 3}
$$

Leading to

$$
10^{77} \leq N_{\text {graviton }}^{4 / 3}
$$

Or an upper bound of say for graviton mass of $10^{-62}$ grams, we have that we have negative pressure in our system for the number of gravitons being less than $10^{58}$, in a volume about 27 times, the cube of Planck length. This is stunning because in Equation (7) we have an entropy number $10^{57}$ to $10^{58}$, which is amazing because it suggests that the entropy generation we pick is tied in explicitly for the generation of negative pressure which is essential for inflation.

\section{Now for How We Could Consider Having $\Delta E$ Drop as Negative Energy, in Our Problem of Pre-Planckian Physics Right before the Onset of Inflation. With A Flip over to Ultra High Temperature-Energy Conditions.}

From [17], we have the following relationship, i.e. see referenced [17] have in its Equation (8) the following value i.e. if $d=$ Dimensions, $P=$ Pressure, $V=$ Volume, then the basic energy expression is given as

$$
E=\frac{d}{2} P V
$$

The discussion as to implementation of Equation (25) has that if the conditions in section 6 above are obtained for negative pressure, that in the Pre Planckian state we have at a chance, a quadratic dispersion relationship. In addition, Reference [17] claims that this is a result of a derivation from the Virial theorem as given in [18], so then that we may look at

$$
\begin{aligned}
& \text { (Heisenberg }) \frac{\mathrm{d} P(\text { momentum })}{\mathrm{d} t}=\frac{i}{\hbar} \cdot[H, P] \\
& \underset{[P, X]=\frac{\hbar}{i} I_{\text {unit matrix }}}{\longrightarrow} \frac{\mathrm{d} P(\text { momentum })}{\mathrm{d} t}=-V^{\prime}(x)
\end{aligned}
$$

This is in turn directly related to the Schrodinger-Ehrenfest theorem we can write as

$$
\frac{\mathrm{d}\langle P(\text { momentum })\rangle}{\mathrm{d} t}=-\left\langle V^{\prime}(x)\right\rangle
$$

This is in a way of referring to [17] and [18] a way to ascertain the correctness of using Equation (25) in the Pre-Planckian to Planckian transition in spacetime. Having said that. We will then state that what we believe is that $V$ as volume, as given in Equation (25) would be roughly about 27 times the cube of Planck length, as a starting point, for investigation and that we would then have a transition up to the Planck length. Prior to nucleation of space-time. 
Our hypothesis is that breaching the barrier to full emergence would entail a simultaneous flip from negative (bound energy states) to Positive energy, whereas we would be using a variant of positive energy given as a restatement of Equation (25)

$$
E(\mathrm{inf})=\frac{d}{2} k_{B} T_{\mathrm{inf}}
$$

i.e. a release of bound state to unrestrained positive energy would be commenced from the Pre Planckian to Planckian transition.

i.e. eventually, if there is a barrier, of space-time at the surface of a sphere of about. 27 times the cube of Plank length, in "volume" that when the barrier was breached, there would be a switch from negative energy, to positive energy, but that the pressure would still be negative, hence "inside" the initial near singularity sphere we would have a negative value of Equation (27) signifying a BOUND state. Once the barrier collapsed, Equation (27) would switch to positive, but that in lieu of inflation that the pressure of our system would still follow Equation (21) and Equation (22).

All this may be tied into an issue of semi classical reasoning as given below. We include this in to motivate readers to consider how a semi classical set of approximations may lead to bridging the gap between General Relativity and Quantum mechanics. We argue that the challenge in our present problem is to reduplicate the same methodology, but to also find a suitable potential system, instead of just a hierarchy of kinetic energy expressions.

\section{Lesson Learned, i.e. a Way to Ascertain if Quantum Gravity Has a Chance to Be Applied Quantum Geometrodynamics and Semi Classical Approximations, as Reference [19] and Evolutionary Equations, for Quantum States, and Its Relationships to Quantum Issues Arising in [20]}

We wish now to refer to another result which we view as largely in tandem with our quest as to come up with precursors to quantum gravity, i.e. from Kieffer.

Due to how huge this literature is, we will be by necessity restricting ourselves to pages 172 to 177 of [19] as that encompasses Hamiltonian style formalism and also has some connections to the Hamilton Jacobi equation.

We will make this limitation so our methods are not too far removed from the Solvay conference, 1927, i.e. the Hamilton-Jacobi equation makes an appearance, as well as a full stationary Schrodinger equation.

In this discussion, the wave functions are often quantized, or nearly so, albeit usually added gravitational background is semi classical.

To begin our inquiry as to Geometrodynamics, which has some fidelity to the Solvay 1927 conference, we look at the following expansion of the Klein Gordon Equation, without an external potential. i.e.

$$
\left(\frac{\hbar^{2}}{c^{2}} \partial_{t}^{2}-\hbar^{2} \Delta+m^{2} c^{2}\right) \Psi_{\text {Klein Gordon }}=0
$$


Which has a series expansion wavefunction solution we can write as

$$
\Psi_{\text {Klein Gordon }}=c^{2} S_{0}+S_{1}+c^{-2} S_{2}+\cdots=\exp \left[i S_{\text {example }} / \hbar\right]
$$

The First, second and third terms in Equation (28a) are as follows and lead to the subsequent Equations, in terms of series expansion powers

$$
S_{0} \text { at } c^{2} \approx \pm m \cdot t \Rightarrow \Psi_{K G}\left(\text { at } c^{2}\right) \approx \exp \left(i c^{2} m \cdot t / \hbar\right)
$$

Then we acquire the term, at no power of $c$, so that

$$
S_{1} \text { at } c^{0} \Rightarrow \Psi_{K G}\left(\text { at } c^{0}\right) \approx \exp \left(i S_{1} / \hbar\right)
$$

Leading to a free Schrodinger equation of the form

$$
i \hbar \partial_{t} \Psi_{K G}\left(\text { at } c^{0}\right)=\frac{-\hbar^{2}}{2 m} \Delta \Psi_{K G}\left(\text { at } c^{0}\right)
$$

Then, the $2^{\text {nd }}$ term of the Klein Gordon Equation in terms of powers of

$$
S_{2} \text { at } c^{-2} \Rightarrow \Psi_{K G}\left(\text { at } c^{-2}\right) \approx \exp \left(i S_{2} / \hbar\right)
$$

Which leads to a Schrodinger like term, with an additional radiation correction term added

$$
i \hbar \partial_{t} \Psi_{K G}\left(\text { at } c^{-2}\right)=\frac{-\hbar^{2}}{2 m} \Delta \Psi_{K G}\left(\text { at } c^{-2}\right)-\frac{h^{4}}{8 m^{3} c^{2}} \Delta \Delta \Psi_{K G}\left(\text { at } c^{-2}\right)
$$

Here we have the following Radiative corrective term added

$$
\frac{h^{4}}{8 m^{3} c^{2}} \Delta \Delta \Psi_{K G}\left(\text { at } c^{-2}\right)
$$

As a Klein Gordon result, this leads directly to the idea of quantum mechanics, as embedded within a larger theory.

i.e. this methodology as brought up by Kieffer, in page 177 of [10] in its own way is fully in sync with some of the investigations of the embedding of quantum mechanics within a larger structure, as has been mentioned in a far more abstract manner by t'Hooft, in [21], although to make further connections, it would be advisable to have a potential term put in, as well as to have more said about relativistic corrections.

As mentioned by [21], Lammerzahl, C. in [22] has extended this sort of reasoning to quantum optics in a gravitational field. The virtue of this is that one is NOT using the functional Schrodinger equation, as seen in page 149 of the Wheeler De Witt equations, given in [19]. i.e. the above derivation, within the context of the orders of $c$, given above, has explicit time dependence put in its evolution equations, and avoids some of the issues of the Wheeler De Witt program. i.e. read page 149 and beyond in [19] as to some of the perils and promises as to this approach.

In addition, the recovery of the Schrodinger equation and the other recovery of the Schrodinger equation with a radiative corrective term added within the context of the Klein Gordon equation is fully in sync with some of the Solvay 1927 deliberations. As given in [20]. And also directly linkable to [21]. 
What we wish to do is to reduplicate the same sort of power expansion picking off of terms given in Equation (28) but instead of using the Klein Gordon Equation, without a potential, to use a similar equation with a potential and from there to ascertain an embedding of space time effects largely in sync with t'Hooft as given in [21] at near the Plank regime of space time. Doing so would among other things employ a redo and looking at how our evolution equation so chosen, as mentioned in Equation (28) may be linked to the issues given in Equation (3) and Equation (4) of our manuscript.

However, before tying an evolution Equation, from Equation (28) suitably modified to use parts of Equation (3) and Equation (4) we need to consider if we have a Hamiltonian system which is the same as the ENERGY of a system. If we do not have this option, it is a good bet that the system so modeled does NOT conserve energy. i.e. What would that mean for our problem?

\section{A Major Caution to Consider, i.e. When We Have a Hamiltonian Which Is Not Conserved, i.e. When Hamiltonian H No Longer Is in Sync with the ENERGY E of a System}

Very simply put, if the Hamiltonian has for any reason a time component to it, so the time derivative of a Hamiltonian is not zero, then the physically modeled system is not conserving energy. i.e. for a $L=L$ (Lagrangian), we have that by [23]

$$
\varepsilon(\text { energy })=\dot{q}_{\beta} \frac{\partial L}{\partial \dot{q}_{\beta}}-L
$$

Whereas we can write if $L=L$ (Lagrangian) has no time dependence, that

$$
\frac{\mathrm{d} \varepsilon(\text { energy })}{\mathrm{d} t}=\frac{\partial L}{\partial t}=0 \text { iff } L=\text { cons } \neq L(t)
$$

The $L=L($ Lagrangian $)=($ Kinetic energy $)-($ Potential energy $)$, hence if we go to look at the Hamiltonian itself we have

$H=H($ Hamiltonian $)=($ Kinetic energy $)+($ Potential energy $)$. Also, we write that the Hamiltonian

$H=H($ Hamiltonian $)=($ Kinetic energy $)+($ Potential energy $)=E($ total energy $)$ if and only if

$$
\frac{\mathrm{d} H}{\mathrm{~d} t}=0 \Rightarrow H(\text { Hamiltonian })=E(\text { Energy })
$$

Otherwise, we have

$$
\frac{\mathrm{d} H}{\mathrm{~d} t} \neq 0 \Rightarrow H(\text { Hamiltonian }) \neq E(\text { Energy })
$$

What we have to decide in terms of the evolution of Equation (3) and Equation (4) is do we have a closed or an open physical input into the creation of the Universe. This will profoundly influence how we look at Equation (20) above, which in turn has a lot to say about how uniformly applicable Equation (24) ac- 
tually is. i.e. if we do this, then there is a matter of the self reproduction of the Universe as given by Mukhanov [24] where we have for a scalar field driving the expansion of the universe, with a scalar field being bigger than the square root of the mass of the universe for domain production as given in [24], page 353.

\section{What if We Wish to Consider Mukhanov Self Reproduction of the Universe Criteria?}

First of all, we will give pertinent background before we go to the Mukhanov criteria.

Note that from [1] [25] we have the following. Note that from [1] [25] we have

$$
a(t)=a_{\min } t^{\gamma}
$$

Leading to [1] the inflaton.

$$
\phi \approx \sqrt{\frac{\gamma}{4 \pi G}} \ln \left\{\sqrt{\frac{8 \pi G V_{0}}{\gamma \cdot(3 \gamma-1)}} \cdot t\right\}
$$

And then we can look at the consequences for self reproduction of the universe, given on page 353 of [24] and its Figure 1 below which is as seen in page 353 , of [24] which is with a perpetuating continual expansion of the universe, given a mass, $m$, for which the scalar field of Equation (34) obeys

$$
\phi>m^{1 / 2}
$$

The results of Equation (35) are accessible in Figure 1 below.

If we use Clifford Will, as in [15] for velocity of a massive graviton and make the following substitutions, we will have if $\gamma \rightarrow \alpha$

$$
\Delta E=N_{g}(\text { \# gravitons }) \cdot m_{g} \cdot c^{2}\left(1-\frac{m_{g}^{2} c^{4}}{\left(E_{\text {system }}=\hbar \omega_{\text {gravitons }}\right)^{2}}\right)
$$

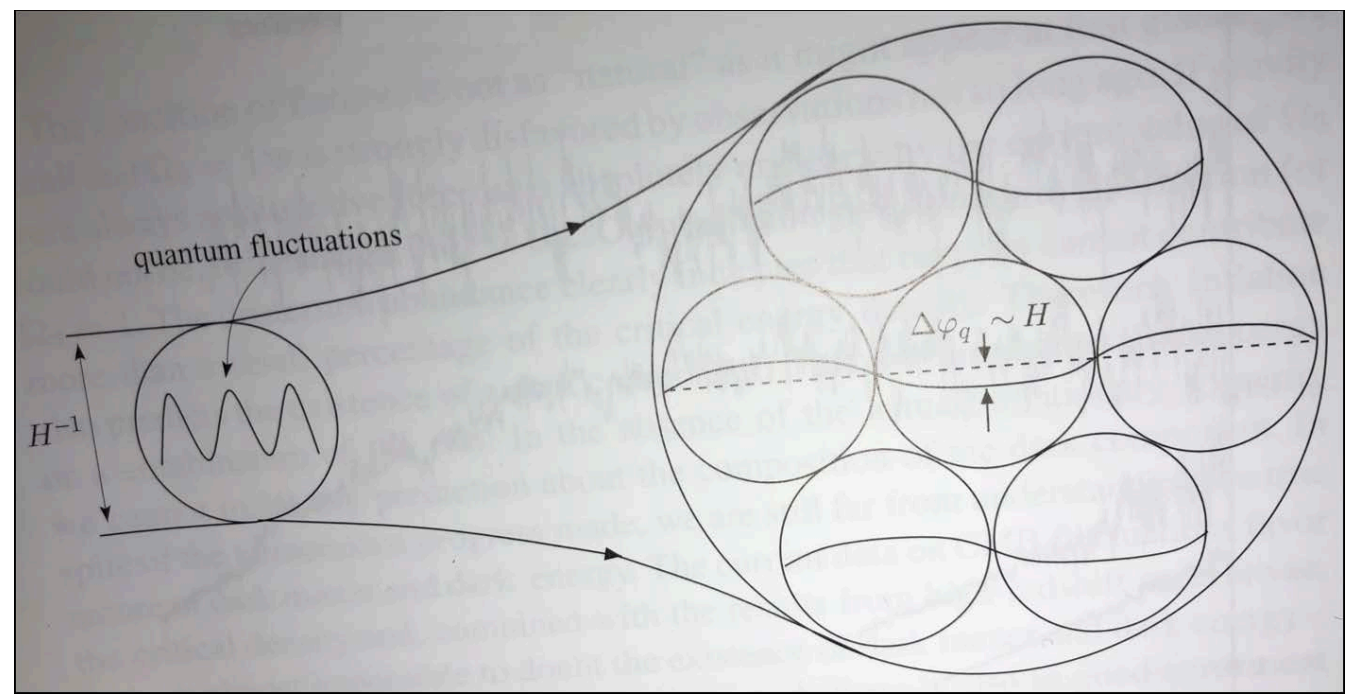

Figure 1. We have in this example a criterion for self reproduction of the universe (based on fig 8.7 of reference [24], i.e. Mukhanov and involving using Equation (35) above. 
This implies (after we set $\hbar=1$ ) with a preliminary minimum time step of $\Delta t=t_{\min }$

$$
\Delta t=\frac{\hbar}{\left(N_{g}(\# \text { gravitons }) \cdot m_{g} \cdot c^{2}\left(1-\frac{m_{g}^{2} c^{4}}{\left(E_{\text {system }}=\hbar \omega_{\text {gravitons }}\right)^{2}}\right)\right)}
$$

Which can be if (after we set $\hbar=1$, and $c=1$ )

$$
\Delta t=\frac{1}{\left(N_{g}(\text { \#gravitons }) \cdot m_{g}\left(1-\frac{m_{g}^{2}}{\left(E_{\text {system }}=\omega_{\text {gravitons }}\right)^{2}}\right)\right)}
$$

Then we have the inequality for self reproduction of the universe as

$$
\sqrt{\frac{8 \pi G V_{0}}{\gamma \cdot(3 \gamma-1)}} \cdot \frac{\hbar}{N_{g} m_{g} c^{2} \cdot\left(1-\frac{m_{g}^{2} c^{4}}{\left(\hbar \cdot \omega_{g}\right)^{2}}\right)} \geq \exp \left(\sqrt{\frac{4 \pi G \cdot N_{g} \cdot m_{g}}{\gamma}}\right)
$$

Also keep in mind the numerical density $N$, as given above, can be linked to a "particle count" due to Entropy.

Then using Kolb and Turner [26], we would see say,

$$
s(\text { Entropy density }) \approx \frac{2 \pi^{2}}{45} \cdot g_{*} \cdot\left(T_{\text {universe }} / T_{\text {Planck }}\right)^{2}
$$

And if we have utilization of $N$ (particle count) $\sim S$ (entropy) as given in [27] by $\mathrm{Ng}$, if we solve conclusively for $N_{g}$ by utilizing Equation (37) we have that, we can rewrite Equation (38) to read as implying

$$
\frac{N_{g}(\text { Graviton count })}{V(\text { volume Planck units })} \approx s(\text { Entropy density }) \equiv \frac{2 \pi^{2}}{45 g^{*}} \cdot\left(T_{\text {Planck temp }} / T_{\text {universe }}\right)^{2}
$$

Should the value $N_{g} \propto 10^{58}$ by earlier arguments in this manuscript, as stated, then if the value of $g_{*} \approx 10^{58} \approx N_{g}$, so then we could have $\left(T_{\text {temperature universe }} / T_{\text {Planck }}\right)^{2} \approx o(1) \simeq 1$, which makes entropy density initially proportional to $g_{*} \approx 10^{58} \approx N_{g}$ will lead to a weird situation later on where one has an infinite (or nearly infinite) number of contributions from parallel or contributing universes from the meta multiverse to start the big bang, in our present universe. In addition, we can look at a shock wave prior to the transition to the inflationary regime that we have the following situation

As given on page 44 of [28]

$$
R_{\text {Shock wave }} \approx v_{\text {shock wave velocity }} \cdot t \equiv \sqrt{E / m} \cdot t
$$

Here the mass is defined by $m \approx \rho \cdot R_{\text {shock wave }}^{3}$. So then

$$
R_{\text {shock wave }}(\text { radius }) \approx(E \cdot t / \rho)^{1 / 5}
$$

This shock wave has to be compared to $\Delta t=\frac{0.845184}{\Delta E}>0$, iff $\Delta E<0$. i.e. 
note that Equation (41) is specifying change in energy, and this can happen before nucleation of the universe, if there is a negative temperature. Which is striking. See also change in scale factor, with change of the temperature of the universe as given in page 401 of [29].

$$
\Delta E \equiv \frac{d(\operatorname{dim})}{2} \cdot k_{B} \cdot T_{\text {universe }}
$$

In doing so, we would then in this case see if we use the real root of $n \sim L / l$, given in Equation (14) above

$$
\Delta t=\left(\frac{0.845154}{d(\operatorname{dim}) \cdot k_{B} \cdot\left|\Delta T_{\text {universe }}\right|}\right)
$$

Then a shock front, right at the starting gate of expansion would look like for the first root of $n \sim L / l$ Equation (14), we then have using Equation (40a)

$$
R_{\text {Shock wave }} \approx\left(\frac{2 \times(0.84545)^{2}}{d(\operatorname{dim}) \times k_{B} \cdot N_{g} \cdot m_{g} \cdot V_{\text {volume Planck units }} \times\left|\Delta T_{\text {universe }}\right|}\right)^{1 / 5}
$$

Here the volume, in this case would be 27 times the cube of Planck length, and the mass of a graviton is approximately $10^{62}$ grams.

\section{Self Reproduction of the Universe May Entail Varying Values of Equation (43) if We Look at Three Roots of $n$ Given in Equation (14), Which Influences a Minimum Time Step}

We state that using the conjugate complex roots of $n \sim L / l$ given in Equation (14) would lead to different values of the numerator of Equation (43) which would lead to different values of Equation (43). We argue that this would induce chaos, and voids in subsequent evolution of space-time. i.e. a matter which we intend to numerically investigate if we have 3 different complementary $n \sim L / l$ values in play used as to Equation (14) and Equation (43).

Keep in mind that if we use the values $m_{\text {Planck }} \equiv 10^{58} m_{\text {graviton }} \equiv \hbar=k_{B}=c=1$ due to renormalization, then Equation (43) becomes if we also assume Planck length scaled to 1 so then we have

$$
R_{\text {Shock wave }} \approx\left(\frac{2 \times(0.84545)^{2}}{d(\operatorname{dim}) \times\left(V_{\text {volume Planck units }} \approx 0.27\right) \times\left|\Delta T_{\text {universe }}\right|}\right)^{1 / 5}
$$

This is obviously semi classical, and we will ask readers to consider that what may be used to add more rigor to our analysis would be the process of Bosonification, as seen in [30], page 319-369 of R. Shankar, with the caveat that we would be considering perhaps using advanced field theory, to have relativistic Dirac Fermions obeying Standard Anti Commutation rules by a Boson field theory. The Fermions would be super partners to the spin two gravitons which in SUSY are spin 3/2 gravitinos.

If SUSY is a non starter, and there have been no confirmed data sets for SUSY 
out of CERN, then we may have to be using gravitons and lump it.

Equation (44) is for the real root of Equation (14). Very likely the two complex roots of Equation (14) would yield different numerator values for the shock wave front formula, and the mixing of all three versions of shock waves, would be itself enough to induce chaos, or at least some of the phenomenology seen in [31]. And if we are lucky in our formulation we may be able to get a potential added to the deliberations of Equation (28), in terms of hierarchy of embedding space time in terms of a power law development. To do that though would require identifying though a suitable potential added, and we need to find that commensurate potential.

\section{More as to a Cosmological Link to the (Weak) Correspondence Principle}

In physics, we have that the correspondence principle is commonly held to be that at large quantum numbers we have an approach to classical results. A request was given to me to quantify that, in terms of mathematics, and the closest which I can come to that is to do the following. I.e. first look at this [32].

Quote

Even if one restricts oneself to Bohr's writings, however, there is still a disagreement among Bohr scholars regarding precisely which of the several relations between classical and quantum mechanics that Bohr discovered should be designated as the correspondence principle. There are three primary candidate-definitions in the literature. First, there is the frequency interpretation, according to which the correspondence principle is a statistical asymptotic agreement between one component in the Fourier decomposition of the classical frequency and the quantum frequency in the limit of large quantum numbers. Second, there is the intensity interpretation according to which it is a statistical agreement in the limit of large quantum numbers between the quantum intensity, understood in terms of the probability of a quantum transition, and the classical intensity, understood as the square of the amplitude of one component of the classical motion. Finally, there is the selection rule interpretation, according to which the correspondence principle is the statement that each allowed quantum transition between stationary states corresponds to one harmonic component of the classical motion.

End of quote

In our situation, we most certainly would prefer the first definition, i.e. to look at.

\section{Quote}

First, there is the frequency interpretation, according to which the correspondence principle is a statistical asymptotic agreement between one component in the Fourier decomposition of the classical frequency and the quantum frequency in the limit of large quantum numbers.

End of quote 
In our situation, a way to illustrate what we are doing is to look at where a large quantum number may play a role as to the approach to classical results. Note in Equation (3) above we have a potential system which we can state the following. Assume that the potential transitions, from Equation (3) where we look at behavior of a "potential" inside and outside a boundary of scale factor space time we write as a boundary we shall call $a_{\text {min }}$

$$
V_{\tilde{N}}(\text { Potential })=0, \text { inside } a_{\text {min }}
$$

Also, we set

$$
V_{\tilde{N}}(\text { Potential })=\tilde{N}, \text { on and outside } a_{\min }
$$

In physics, the correspondence principle states that the behavior of systems described by the theory of quantum mechanics (or by the old quantum theory) reproduces classical physics in the limit of large quantum numbers.

What we are doing is to assume a large quantum number will be generated just about the transition from the interior to the exterior of $a_{\min }$.

As it is, I expect that the transition of steps given in Equation (45) and Equation (45a) will lead to the following, i.e. as we have the transition from small to large values of a potential given in Equation (3) as stated in Equation (45) and Equation (45a) we would then have the following as given by Pauli, [33] on page 33. We take the spin zero result since it is a BOSON and assume a similar qualitative overlap with spin 2 gravitons. Now we have that we can evaluate Equation (3) in terms of the Vacuum energy, and this is what we get if the quantum numbers get enormous. i.e. the cosmological constant approaches the value of Vacuum energy as given by Pauli, for Bosons, and we will take some approximations. From Equation (3) and also from Pauli for Bosons [33]

$$
\Lambda \approx \text { Vacuum energy } \equiv\left(\frac{1}{2 \pi}\right)^{3} \cdot \int_{0}^{k \text { (critical value })} k^{2} \cdot \sqrt{k^{2}+m^{2}} \mathrm{~d} k \simeq\left(\frac{1}{2 \pi}\right)^{3} \times\{\}
$$

Where we get from Pauli, [33] that if we have large quantum numbers inside the well, we have

$$
\Lambda \approx \text { Vacuum energy } \approx\left(\frac{1}{2 \pi}\right)^{3} \times\left\{\frac{k_{\text {critical value }}^{4}+m^{2} k_{\text {critial value }}^{2}-m^{4} \ln \left(\frac{2 k_{\text {critial value }}}{m}\right)}{4}\right\}
$$

So we do not have a complete break down of our results we assume here the following substitutions, that

$$
k(\text { critical }) \rightarrow p(\text { momentum })
$$

Furthermore, we have then that if we use the speed of a massive graviton as given by [34], i.e. if $\hbar=c=1$ and $m \rightarrow m_{g}$, an $\Delta E \Delta t=1$. Then the vacuum energy would be for Equation (46) approximately if we used a large quantum number for Equation (3) for the interior region approaching, and if we have 
momentum defined by a velocity, as $v_{\text {graviton mass }} \approx\left(1-m_{g}^{2} \Delta t^{2}\right)^{1 / 2}$, if $c=1$, so then we have a vacuum energy

$$
\text { Vacuum energy } \approx m_{g}^{3} \cdot\left(1-m_{g}^{2} \Delta t^{2}\right)^{3 / 2} \cdot\left(m_{g}^{2} \cdot\left(1-m_{g}^{2} \Delta t^{2}\right)+m_{g}^{2}\right)^{1 / 2}
$$

i.e. and this gets into one of the issues brought up by Christian Corda who asked about it. i.e. is there a way to reconcile the value of a cosmological constant as given by Wesson, in 5-dimensional cosmology with that of what is in official data sets. Before going to this issue, we should consider [35]

$$
\text { Vacuum energy } \approx \frac{\Lambda}{8 \pi G}
$$

As we have a value of minimum time step $\Delta t$ from Equation (36) above, we can then conflate what we are doing with Wesson, i.e. what we did is to assume that there was a projection of space-time from 5 dimensions onto four dimensions i.e. according to this metric as given by Wesson [36], i.e. see its page 44 Equation (2.42)

$$
\mathrm{d} S^{2}=\left(\frac{l^{2}}{L^{2}}\right) \cdot\left[\left(1-\frac{2 M}{r}-\frac{r^{2}}{L^{2}}\right) \mathrm{d} t^{2}-\frac{\mathrm{d} r^{2}}{\left(1-\frac{2 M}{r}-\frac{r^{2}}{L^{2}}\right)}-r^{2} \mathrm{~d} \Omega^{2}\right]-\mathrm{d} l^{2}
$$

The terms in the brackets refer to a 3-dimensional space, with four-dimensional time component, whereas $\mathrm{d} l^{2}$ is for the $5^{\text {th }}$ dimension. In this context, the cosmological constant, is then according to [36], assuming that $L$ is for fourdimensional Space-time

$$
\Lambda \equiv 3 / L^{2}
$$

A word of explanation is due here. What I assumed in the calculation of $\Delta t$, in terms of time step is to look at a projection and interaction of the fourth and $5^{\text {th }}$ dimensions to come up with the MININUM time step, and then from there to insert it into Equation (48).

Our working assumption is as follows, i.e. that what we have, as of Equation (48) should be virtually identical in magnitude to Equation (51) but it should be understood that $\mathrm{L}$ in Equation (51) is really the present day value of the assumed "radius of the universe". i.e. we are assuming then from Pre Planckian conditions to our present day that the Cosmological constant does not change. In any case, the approximate value of the Cosmological constant in Equation (51) should be understood to be by observations, approximately as follows, i.e. the true dimension of $\Lambda \propto 1 /(\text { Length })^{2}$. With the following sort of Dimensional magnitude as given below.

$$
\begin{aligned}
\Lambda & \propto 1 /(\text { Length })^{2} \approx 4.33 \times 10^{-60}(\mathrm{eV}=\text { Electron Volt })^{2} \\
& \equiv 2.88 \times 10^{-122}(\text { Planck Units })
\end{aligned}
$$

We can get some of the observational thinking as to measurements of this constant, via [37], which is from Supernova candle results, so finally as brought 
up by Christian Corda, there is a matter of connecting the Pre-Planckian with Planckian results [38], which is the backbone of the delta $t$ term used in Equation (48)

\section{More on a Linkage to Pre-Planckian to Planckian Physics}

One of the striking results in [38] is their treatment of entropy, as given in their Equation (40), which is brought up to take into consideration the possibility of tunneling. i.e. the variation in entropy, $\Delta S$, is given as

$$
i \Delta S=\frac{i k E t}{\hbar}-\frac{E}{t}
$$

My first conclusion is that if there is a tie into the formula 27 of my manuscript that in fact what was done in [38] may be a way to tie in energy, $E$, with entropy, and make the analogy to Tunneling from the interior to the exterior of a boundary between pre Planckian to Planckian space time more exact.

I would be inclined to take the absolute magnitude of this above entropy expression and to assume the following, i.e. in the aftermath of tunneling right at the nexus of a boundary we would see approximately have for entropy generation, using the absolute magnitude of [38] as well as delta $S \sim n$ (particle counting) by infinite quantum statistics as given by $\mathrm{Ng}$. [39]. An advantage of Equation (52) if confirmed would be a way to examine the Weak correspondence principle more exactly. We shall comment upon this in our conclusion. Here we take the absolute value of Equation (52) and we will use that in our conclusion.

$$
|\Delta S|=\sqrt{\left|\frac{k E t}{\hbar}\right|^{2}+\left|\frac{E}{t}\right|^{2}}=\text { particle count } .
$$

\section{Conclusion: Part A: Can We Use Equation (52) and Equation (53) to Quantify a Correspondence Principle in Cosmology Precisely?}

I wish to Thank Christian Corda for bringing this question to my attention. The answer is maybe, but if we do that we can assume that the modeling of $E$, used in Equation (53) may be commensurate for the energy levels of a spherical infinite square well, i.e. see this [40]. We will assume the spherical, zero angular momentum case if we do this, so then we have if the radius of the well has zero inside the well and an infinite potential barrier value just outside, that to first approximation we have that by [40].

$$
E_{n, 0}=\frac{n_{\text {quantum }}^{2} \pi^{2} \hbar^{2}}{2 m \cdot r_{\text {spherical well }}^{2}}
$$

My off the top of my head idea is to compare the value of Equation (54) with the value of Equation (27) which has an explicit Temperature dependence. Making the approximation that $\mathrm{m}$, in this last set of calculation is the same as 
the mass of a graviton, and that the term a, as given above is less than or equal to Planck length, if the resulting $n$, as used in Equation (54) is large, and ties in with Equation (27), with that temperature dependence, we may see the start of classical to quantum correspondence, for large n, and a tie in that way to the Weak correspondence principle. What we can do is to look also at a relation given by Kerson Huang, in [41], as well as page 481 of the Hubble parameter given in [42] where we have normalized the Planck mass to have a value of 1 . If so then

$$
\frac{8 \pi G \rho}{3 \tilde{H}(\text { Hubble parameter })^{2}}-\frac{\kappa_{\text {Flatness parameter }}}{\tilde{H}(\text { Hubble parameter })^{2} \cdot a(t)^{2}}=1
$$

If so, then we can look at

$$
\kappa_{\text {Flatness parameter }}=-\left(1.66 \times \sqrt{g_{*}} \cdot T_{\text {max temperature }}^{2}\right)^{2} \cdot a(t)^{2}+8 \pi G \rho \times a(t)^{2}
$$

If we use the value of $a(t)=a_{\min } t^{\gamma}$, and then we have

$$
\kappa_{\text {Flatness parameter }}=\left[-\left(1.66 \times \sqrt{g_{*}} \cdot T_{\text {max temperature }}^{2}\right)^{2}+8 \pi G \rho\right] \times a_{\min }^{2} t^{2 \gamma}
$$

In order for this Equation (56) to be greater than equal to zero, we would need to have

$$
8 \pi G \rho \geq(1.66)^{2} g_{*} T_{\mathrm{Temp}}^{4}
$$

How to tie this into the matter of energy. i.e. use for Pre Planckian to Planckian transitions showing large quantum number values so that the correspondence principle in cosmology would hold would be to have used energy as given in Equation (54)

$$
\left.n^{2} \text { (quantum number }\right)=\left[\frac{2 \cdot V_{\text {space volume }} \cdot m_{g}\left(1.66 \times \sqrt{g_{*}} \cdot T_{\text {max temperature }}^{2}\right)^{2}}{\pi^{2} \hbar^{2}}\right]
$$

We should before proceeding also note that we would also be utilizing having Equation (41) so that we have,

$$
n^{2}(\text { quantum number }) \approx \frac{2 d(\operatorname{dim}) \cdot V_{\text {space volume }} m_{g} \cdot\left(R_{\text {radius well }}\right)^{2}}{\pi^{2} \hbar^{2}}
$$

Where we are assuming having an almost one to one connection between $g_{*}$ and $d(\operatorname{dim})$

$$
\rho=\frac{E(\text { cylindrical well energy })}{V_{\text {Volume }}}=\frac{\left.n^{2} \text { (quantum number }\right) \cdot \pi^{2} \hbar^{2}}{V_{\text {Volume }} \cdot m_{g} \cdot\left(r_{\text {radius well }}\right)^{2}}
$$

For there to be an equality, which would be a necessary condition for having a defacto correspondence principle in Cosmology, i.e. to have quantum effects for high numbers, i.e. $n_{\text {quantum number }}^{2} \gg 1$, one would likely have, even if we state $g_{*}$ is a degree of freedom, would be that the stated dimensional values of inputs into a very large value for $d_{\text {dim }}$ for inputs into the Pre Planckian state, prior to 
emergence into Planckian cosmology conditions would have to be an extremely large number. i.e. we would be looking for conditions in the pre-Planckian space time for which $n_{\text {quantum number }}^{2} \gg 1$ due to an enormous value for $d_{\text {dim }}$.

In saying this, we have to be more precise than we have been wont to be in geometry of pre-Planckian space time. And if $n_{\text {quantum number }}^{2}$ approached 1, for whatever the reason, the chances that we could evaluate Equation (53) in terms of the Correspondence principle would evaporate.

\section{Conclusion, Part B: Can Extra Dimensions Come from a Multiverse Feed into Pre-Planckian Space-Time? See Theorem}

To do this what we do is to state the multiverse done in [43] and [44] and cite the number, $\mathrm{N}$ so brought up with changes in $g_{*}$, which is, the degree of freedom so assumed.

This idea is extremely speculative, but it embodies using this version of an idea which is in a recent conference proceedings in Spain used these two references [43] [44].

i.e. the DNA of the idea was to refer to a Multiverse version of what is known as the Penrose Cyclic Conformal cosmology conjecture, i.e. [45] use this construction.

We are extending Penrose's suggestion of cyclic universes, black hole evaporation, and the embedding structure our universe is contained within, This multiverse embeds BHs and may resolve what appears to be an impossible dichotomy. The following is largely taken from [43] and [44] and has serious relevance to the final part of the conclusion. That there are no fewer than $N$ universes undergoing Penrose "infinite expansion" (Penrose) [45] contained in a mega universe structure. Furthermore, each of the $N$ universes has black hole evaporation, with the Hawking radiation from decaying black holes. If each of the $N$ universes is defined by a partition function, called $\left\{Z_{i}\right\}_{i=1}^{n}$ then there exist an information ensemble of mixed minimum information correlated as about $10^{7}$. $10^{8}$ bits of information per partition function in the set $\left.\left\{Z_{i}\right\}_{i=1}^{n}\right|_{\text {Before }}$, so minimum information is conserved between a set of partition functions per universe

$$
\left.\left.\left\{Z_{i}\right\}_{i=1}^{n}\right|_{\text {Before }} \equiv\left\{Z_{i}\right\}_{i=1}^{n}\right|_{\text {After }}
$$

However, there is non-uniqueness of information put into each partition function $\left\{Z_{i}\right\}_{i=1}^{n}$. Furthermore Hawking radiation from the black holes is collated via a strange attractor collection in the mega universe structure to form a new big bang for each of the $N$ universes represented by $\left\{Z_{i}\right\}_{i=1}^{n}$. Verification of this mega structure compression and expansion of information with a non-uniqueness of information placed in each of the $N$ universes favors ergodic mixing treatments of initial values for each of $N$ universes expanding from a singularity beginning. The $S$ (entropy) $\sim n_{f}$ value, will be using $(\mathrm{Ng}, 2008)$ $S$ (entropy) $\sim n_{f}$ [28]. How to tie in this energy expression, will be to look at the 
formation of a nontrivial gravitational measure as a new big bang for each of the $N$ universes as by $n\left(E_{i}\right)$ the density of states at a given energy $E_{i}$ for a partition function. (Poplawski, 2011) [46]

$$
\left\{Z_{i}\right\}_{i=1}^{n} \propto\left\{\int_{0}^{\infty} \mathrm{d} E_{i} n\left(E_{i}\right) \cdot \exp \left(-E_{i}\right)\right\}_{i=1}^{N}
$$

Each of $E_{i}$ identified with Equation (61) above, are with the iteration for $N$ universes (Penrose, 2006) [45] Then the following holds, by asserting the following claim to the universe, as a mixed state, with black holes playing a major part, due to the CCC cosmological picture, by starting off with

\section{Claim 1}

$$
\left.\left.\frac{1}{N} \cdot \sum_{i=1}^{N}\left\{Z_{i}\right\}\right|_{i \text { Before nucleation regime }} \underset{\text { Affer Planck era }}{\longrightarrow}\left\{Z_{i}\right\}\right|_{i \text { fixed After nucleation regime }}
$$

For $N$ number of universes, with each $\left.\left\{Z_{i}\right\}\right|_{i \text { Before nucleation regime }}$ for $i=1$ to $N$ being the partition function of each universe just before the blend into the RHS of Equation (62) above for our present universe. Also, each of the independent universes given by are constructed by the absorption of one to ten million black holes taking in energy. i.e. (Penrose) [45]. Furthermore, the main point is similar to what was done in [47] in terms of general ergodic mixing The second Claim is that the dynamics of black holes, in a particular universe, call it, the ith, one, are of critical importance.

Claim 2

$$
\left.\left.\left\{Z_{i}\right\}\right|_{i \text { Before nucleation regime }} \approx \sum_{k=1}^{\text {Maximum }}\left\{Z_{k}\right\}\right|_{k \text { Black-Hole nucleation regime }}
$$

What is done in Claim 1 and Claim 2 is to come up with a protocol as to how a multi-dimensional representation of black hole physics enables continual mixing of spacetime [47] largely as a way to avoid the Anthropic principle, as to a preferred set of initial conditions.

What this Ergodic condition of mixing of different contributions in the Pre Planckian space-time would do is to add, via using up to $N$ (almost infinite, say) multiverse contributions to a CCC version of space time is to add a statistical averaging of an initial start from a Pre Planckian to Planckian transition.

Prior to working with the theorem, we wish to bring up the following, i.e. that we would write.

The number, $N$ of different multiverse contributions to a pre Planckian space-time would then lead to the following theorem.

\section{Space-time dimensional Theorem (involving ergodic mixing)}

The number of multiverse contributions, call it $N$ (number of multiverse contributions) has a 1-1 relationship to the coefficient, $\mathrm{d}(\mathrm{dim})$ as of an equality in Equation (58) and Equation (59) so that the quantum number obtained in the left hand side of Equation (58) and also Equation (59) will be sufficiently large to permit values $\gg 1$ such that the quantum version of quantum gravity linked to classical GR holds after the transition to from Pre Planckian to Planckian physics 
commences.

Proof

First of all write

$$
g_{*}=N(\text { multiverse number }) \times g_{*}(\text { individual universe })
$$

Here, we will define, $g_{*}$ (individual universe) in terms of what is given in Kolb and Turner [27], see that usually [27] has a value of, in the very early universe, of $g_{*}=102$ d.o.f. i.e. 102 degrees of freedom (for each individual universe), i.e, i.e. if one is using Equation (64) we then conclude with writing

$$
\begin{aligned}
n_{\text {quantum number }}^{2} \equiv & \frac{2 \cdot V_{\text {space volume }} m_{g} \cdot\left(R_{\text {radius well }}\right)^{2} \cdot(1.66)^{2}}{\pi^{2} \hbar^{2}} \\
& \cdot N_{\text {Contributing universes }} \cdot g_{* \text { individual universe }} \cdot T_{\text {universe }}^{4}
\end{aligned}
$$

Concluding that we can state directly that.

$d_{\text {dim }}$ varies directly with $N$, where $N$ is the number of individual multiverse components (66).

We furthermore state that this procedure, as similar to a black hole (not identical) and, has much overlap with Dr. George Chapline's et al. [48].

If this theorem is upheld as far as being proven, a road to quantum, gravity exists. This idea will be significantly developed in future publications.

Chapline, et al., state as follows on page 1 of their article [48].

Quote

The black hole event horizon is a continuous quantum phase transition of the vacuum of space-time roughly analogous to the quantum liquid-vapor critical point of an interacting bose fluid.

End of quote

We are doing much the same sort of thing, in the Pre Planckian to Planckian transition, and we will add far more detail relevant to experimental confirmation in a future article follow up which conceivably could be tested via experimental.

\section{Acknowledgements}

This work is supported in part by National Nature Science Foundation of China grant No. 11375279.

Personal big thank you to Dr. Christian Corda for his outstanding questions which are highlighted in this manuscript.

\section{Conflicts of Interest}

The author declares no conflicts of interest regarding the publication of this paper.

\section{References}

[1] Padmanabhan, T. (2006) An Invitation to Astrophysics. World Scientific Series in Astronomy and Astrophysics: Volume 8, World Press Scientific, Singapore. https://doi.org/10.1142/6010 
[2] Diosi, L. (2019) Planck Length Challenges of Non Relativistic Quantum Mechanics of Large Masses. Journal of Physics. Conference Series, 1275, Article ID: 011001. https://arxiv.org/abs/1903.04852 https://doi.org/10.1088/1742-6596/1275/1/012007

[3] Beckwith, A. (2018) Using "Enhanced Quantization" to Bound the Cosmological Constant, (for a Bound-On Graviton Mass), by Comparing Two Action Integrals (One Being from General Relativity) at the Start of Inflation.

http://vixra.org/pdf/1802.0305v1.pdf

[4] Klauder, J. (2015) Enhanced Quantization, Particles, Fields \& Gravity. World Press Scientific, Singapore. https://doi.org/10.1142/9452

[5] Beckwith, A. (2016) Gedanken Experiment for Refining the Unruh Metric Tensor Uncertainty Principle via Schwarzschild Geometry and Planckian Space-Time with Initial Nonzero Entropy and Applying the Riemannian Penrose Inequality and Initial Kinetic Energy for a Lower Bound to Graviton Mass (Massive Gravity). Journal of High Energy Physics, Gravitation and Cosmology, 2, 106-124. https://doi.org/10.4236/jhepgc.2016.21012

[6] Giovannini, M. (2008) A Primer on the Physics of the Cosmic Microwave Background. World Press Scientific, Hackensack. https://doi.org/10.1142/6730

[7] Beckwith, A. (2017) Creating a (Quantum?) Constraint, in Pre Planckian SpaceTime Early Universe via the Einstein Cosmological Constant in a One to One and onto Comparison between Two Action Integrals. (Text of Talk for FFP 15, Spain November 30, 11 Am-11:30 Am, Conference). http://vixra.org/abs/1711.0355

[8] Camara, C.S., de Garcia Maia, M.R., Carvalho, J.C. and Lima, J.A.S. (2004) Nonsingular FRW Cosmology and Non Linear Dynamics. Physical Review D: Particles and Fields, 69, Article ID: 063501. https://doi.org/10.1103/PhysRevD.69.123504

[9] Novello, M. (2005) The Mass of the Graviton and the Cosmological Constant Puzzle. https://arxiv.org/abs/astro-ph/0504505

[10] https://www.mpi-hd.mpg.de/lin/events/group_seminar/inflation/heeck.pdf

[11] Corda, C. (2018) Space-Time Can Be Neither Discrete Nor Continuous. Modern Physics Letters A, 33, Article ID: 1850069. https://doi.org/10.1142/S0217732318500694

[12] Wesson, P. (2006) Five Dimensional Physics, Classical and Quantum Consequences of Kaluza-Klein Cosmology. World Press Scientific, Singapore.

[13] CRC (1969) Standard Mathematical Tables. 17th Edition, Chemica Rubber Co., Cleveland.

[14] Volovik, G.E. (2003) The Universe in a Helium Droplet. Oxford Science Publications, New York.

[15] Will, C. (2018) Theory and Experiment in Gravitational Physics. 2nd Edition, Cambridge University Press, New York.

[16] NIST (2019) 2018 CODATA Value: Planck Mass. The NIST Reference on Constants, Units, and Uncertainty.

[17] Giuseppe, F.M. and Trombettoni, M.A. (2014) Energy-Pressure Relation for LowDimensional Gases. Nuclear Physics B, 887, 216-245. https://doi.org/10.1016/j.nuclphysb.2014.08.007

[18] Messiah, A. (1999) Quantum Mechanics, Two Volumes Bound as One. Dover Publications, Inc., Mineola.

[19] Kieffer, C. (2012) Quantum Gravity. 3rd Edition, Oxford Science Publications, Oxford University Press, Oxford. 
[20] Bacciagaluppi, G. and Valentini, A. (2009) Quantum Theory at the Crossroads: Reconsidering the 1927 Solvay Conference. Cambridge University Press, Cambridge.

[21] t'Hooft, G. (2002) Determinism beneath Quantum Mechanics. Proceedings Quo Vadis Quantum Mechanics, Philadelphia, 12 p. https://arxiv.org/abs/quant-ph/0212095

[22] Lammerzahl, Z.C. (1995) A Hamiltonian Operator for Quantum Optics in Gravitational Fields. Physics Letters A, 203, 23-27. https://doi.org/10.1016/0375-9601(95)00345-4

[23] Kompaneyetes, A.S. (1962) Theoretical Physics. Dover Publications, New York.

[24] Mukhanov, V. (2005) Physical Foundations of Cosmology. Cambridge University Press, New York. https://doi.org/10.1017/CBO9780511790553

[25] Beckwith, A.W. (2018) Using "Enhanced Quantization" to Bound the Cosmological Constant, and Computing Quantum Number n for Production of 100 Relic Mini Black Holes in a Spherical Region of Emergent Space-Time. Journal of High Energy Physics, Gravitation and Cosmology, 4, 549-566. https://doi.org/10.4236/jhepgc.2018.43033

[26] Kolb, E. and Turner, M. (1994) The Early Universe. Avalon Publishing, New York.

[27] Ng, Y.J. (2008) Spacetime Foam: From Entropy and Holography to Infinite Statistics and Nonlocality. Entropy, 10, 441-461. https://doi.org/10.3390/e10040441

[28] Ostriker, J.P. (1991) Development of Large Scale Structure in the Universe. Press Syndicate of the University of Cambridge, New York.

[29] Collins, P.D., Martin, A.D. and Squires, E.J. (1989) Particle Physics and Cosmology. Wiley Interscience, of John Wiley and Sons, New York.

[30] Shankar, R. (2019) Quantum Field Theory and Condensed Matter, an Introduction. Cambridge University Press, New Deli.

[31] Ruutu, V., Eltsov, V., Gill, A., Kibble, T., Krusius, M., Makhlin, Y.G., Placais, B., Volvik, G. and Wen, Z. (1996) Vortex Formation in Neutron-Irradiated ${ }^{3} \mathrm{He}$ as an Analog of Cosmological Defect Formation. Nature, 382, 334-336.

https://doi.org/10.1038/382334a0

[32] https://plato.stanford.edu/entries/bohr-correspondence

[33] Pauli, W. (1981) Pauli Lectures on Physics Volume 6 Selected Topics in Field Quantization. The MIT Press, Cambridge.

[34] Maggiore, M. (2008) Gravitational Waves, Volume 1: Theory and Experiment. Oxford University Press, New York.

[35] Sola, J. (2013) Cosmological Constant and Vacuum Energy: Old and New Ideas. Journal of Physics: Conference Series, 453, Article ID: 012015.

https://arxiv.org/abs/1306.1527v3 https://doi.org/10.1088/1742-6596/453/1/012015

[36] Wesson, P. and Overduin, J. (2019) Principles of Space-Time-Matter, Cosmology, Particles, and Waves in 5 Dimensions. World Scientific Publishing Co., Singapore.

[37] Carmeli, M. and Kuzmenko, T. (2001) Value of the Cosmological Constant: Theory versus Experiment. https://cds.cern.ch/record/485959/files/0102033.pdf

[38] Feleppa, F., Licata, I. and Corda, C. (2019) Hartle-Hawking Boundary Conditions as Nucleation by de Sitter Vacuum. Physics of the Dark Universe, 26, Article ID: 199381. https://arxiv.org/abs/1909.07824 https://doi.org/10.1016/j.dark.2019.100381

[39] Ng, Y.J. (2008) Space-Time Foam: From Entropy and Holography to Infinite Statis- 
tics and Nonlocality. Entropy, 10, 441-461.

https://doi.org/10.3390/e10040441

[40] https://www.physics.wisc.edu/undergrads/courses/fall09/205/lecture_notes/chapter 07.pdf

[41] Huang, K. (2017) A Superfluid Universe. World Scientific Publishing Co., Singapore.

[42] Sarkar, U. (2008) Particle and Astroparticle Physics. Taylor and Francis Series in High Energy Physics, Cosmology, and Grasvitation, New York.

[43] Hertzberg, M.P. and Yamada, M. Primordial Black Holes from Polynomial Potentials in Single Field Inflation.

[44] Beckwith, A. (2014) Analyzing Black Hole Super-Radiance Emission of Particles/Energy from a Black Hole as a Gedanken Experiment to Get Bounds on the Mass of a Graviton. Advances in High Energy Physics, 2014, Article ID: 230713. https://arxiv.org/abs/1404.7167

[45] Penrose, R. (2011) Cycles of Time-An Extraordinary New View of the Universe by Alfred A. Knopf, New York.

[46] Poplawski, N. (2011) Cosmological Constant from QCD Vacuum and Torsion. Annals of Physics (Berlin), 523, 291. https://doi.org/10.1002/andp.201000162

[47] Dye, H. (1964) On the Ergodic Mixing Theorem. http://www.ams.org/journals/tran/1965-118-00/S0002-9947-1965-0174705-8/S00029947-1965-0174705-8.pdf

[48] George, C., Hohlfeld, E., Laughlin, R.B. and Santiago, D.I. (2001) Quantum Phase Transitions and the Breakdown of Classical General Relativity. Philosophical Magazine Part B, 81, 235. https://doi.org/10.1080/13642810108221981 